\title{
Variabilidad de los Efectos de la Motivación sobre las Estrategias Desarrolladas por Ratas en el Laberinto Radial
}

\section{Variability of food motivation effects on the strategies developed by rats in the radial maze}

\author{
Fabián Soto Caro* \\ María de los Ángeles Saavedra**
}

\begin{abstract}
Resumen
Investigaciones previas han mostrado que el nivel de deprivación alimenticia influiría sobre el tipo de estrategia utilizada por ratas en el laberinto de brazos radiales. Los animales más motivados utilizarían estrategias basadas en claves egocéntricas propioceptivas, mientras los menos motivados utilizarían estrategias basadas en claves alocéntricas visuales. En la presente investigación se intentó replicar tales resultados con el objeto de explorar más finamente estas estrategias. Sin embargo, se obtuvieron resultados negativos en dos experimentos. En el experimento 1, 24 ratas albinas fueron separadas en dos grupos con distinto peso corporal: los animales en el grupo G75 $(n=12)$ fueron deprivados de comida hasta alcanzar un $75 \%$ de su peso de alimentación libre, y los animales en el grupo G85 ( N=12) fueron deprivados hasta alcanzar un $85 \%$ de su peso de alimentación libre. En el experimento 2, 9 ratas albinas fueron separadas en dos grupos con distintas raciones diarias de comida: $\mathrm{GB}(\mathrm{n}=5)$ con una ración de 10 gramos, y GA $(n=4)$ con una ración de 20 gramos. No se encontraron diferencias significativas en el uso de estrategias en ninguno de los dos experimentos. Estos resultados cuestionan la generalidad de la relación previamente encontrada entre el nivel de motivación de las ratas y el uso de estrategias para la búsqueda de comida en el laberinto radial. Se discute acerca de las condiciones específicas bajo las cuales un mayor nivel de deprivación alimenticia llevaría a utilizar estrategias más simples, basadas en claves propioceptivas, para abastecerse de comida en el laberinto radial.
\end{abstract}

Palabras claves: laberinto radial, estrategias, deprivación de comida, motivación

\begin{abstract}
Previous research had shown that the level of food deprivation determines the type of strategy used by rats in the radial arm maze. Highly motivated rats use strategies based on proprioceptive cues while the less motivated animals use strategies based
\end{abstract}

* Laboratorio de Psicología Experimental, Universidad La República

** Universidad de Chile, Departamento de Psicología, Facultad de Ciencias Sociales 
on visual-distal cues. The purpose of the present study was to replicate these findings to further explore these strategies. However, negative results were obtained in two experiments. In experiment 1 , twenty four albino rats were divided in two groups with different levels of food deprivation: animals in group G75 $(n=12)$ were fooddeprived until they reached $75 \%$ of their initial weight, and animals in group G85 until they reached $85 \%$ of its normal weight. In experiment 2, 9 albino rats were divided into two groups to reach similar weight differences with a different feeding procedure: GB received 10 grams per day and GA 20 daily grams. No differences in strategies were observed under either condition. These results question the generality of the relation previously encountered between food deprivation level and the use of different strategies to solve the radial maze task. Results are discussed in terms of the specific conditions that would lead to the use of simpler strategies -based in proprioceptive cues- to obtain food in the radial arm maze.

Key words: radial maze, strategy, food deprivation, motivation

Desde su creación por Olton y Samuelson (1976), el laberinto de brazos radiales ha sido utilizado extensamente en estudios de memoria espacial en animales, lo que incluye estudios acerca de los factores sensoriales (Foreman y Stevens, 1982), neurofisiológicos (para revisiones, ver Good, 2002; Macphail, 2002; Poucet y Benhamou, 1997), farmacológicos (e.g., Watts, Stevens y Robinson., 1981; Ammassari-Teule y Caprioli, 1985) y etáreos (e.g., Hall y Berman, 1995) de este tipo de memoria. Este laberinto consiste en 8 brazos idénticos que se proyectan radialmente desde una plataforma central (ver fig. 1). La tarea de los sujetos expuestos a esta situación implica recorrer cada brazo del laberinto, sin repetir ninguno, para recoger refuerzos ubicados en sus extremos. La ejecución en el laberinto permite evaluar la memoria de trabajo espacial en roedores, quienes deberían recordar en cada nueva elección los brazos previamente visitados para no cometer un error. La estrategia de respuesta estaría basada en el aprendizaje previo acerca de la relación entre claves distales o alocéntricas del ambiente y cada uno de los brazos del laberinto, lo que permitiría identificar las avenidas ya visitadas. Esto sin importar la forma en que los animales lleguen a tal conocimiento, asunto que es cuestión de debate aún actualmente (ver Bennett, 1996; Chamizo, 2002, 2003; Gallistell, 1993; para la discusión actual acerca de si el aprendizaje de claves alocéntricas es asociativo o basado en la construcción de un "mapa cognitivo").

Figura 1: Laberinto de brazos radiales de Olton y Samuelson (1976). En la parte di stal de cada avenida seencuentra una hendidura que permite contener un grano de arroz inflado, sin que pueda ser observado por el animal desde ed centro de la plataforma.

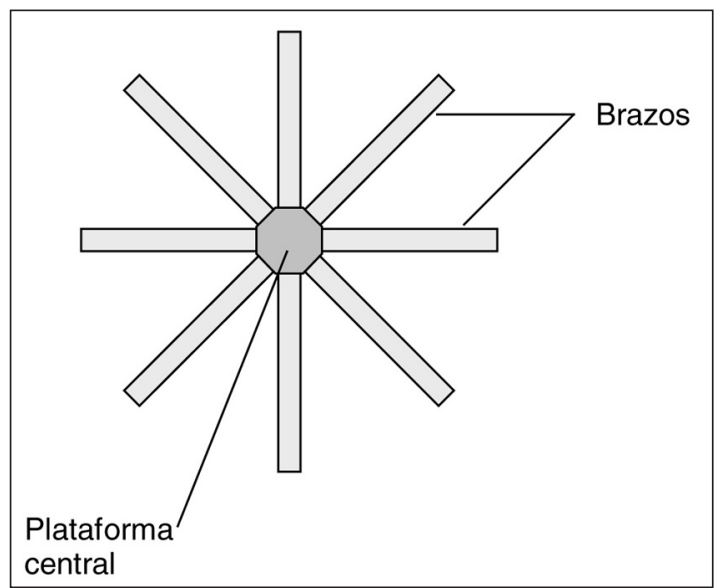

Uno de los aspectos más ampliamente investigados con respecto al comportamiento de roedores en el laberinto radial es la medida en que éstos utilizan estrategias que les permiten simplificar la tarea, evitando el uso de funciones cognitivas superiores como la memoria de trabajo y representaciones elaboradas de su entorno espacial. Olton, 
Collison y Werz (1977) lograron identificar un patrón de respuestas que reconocerían como simplificador de la tarea, consistente en escoger sucesivamente brazos en el sentido (o contra el sentido) de las agujas del reloj, saltando al menos un brazo (es decir, escogiendo el siguiente brazo al menos a $90^{\circ}$ del previamente escogido) en cada opción. Luego se determinaría que la estrategia más frecuente no implica saltar ningún brazo en cada nueva elección, sino elegir el primer brazo aleatoriamente y luego consistentemente el brazo adyacente a aquél del cual se está saliendo, hasta completar los ocho brazos y terminar de recoger todos los refuerzos. Tal estrategia se encontraría basada en el uso de claves egocéntricas-propioceptivas, y tal como ha planteado Eckerman (1980), no implica el uso de memoria espacial de trabajo o de aprendizaje de claves alocéntricas, debido a que no se elige los brazos en base a si la comida ha sido o no removida previamente, sino que se utiliza una regla general del tipo "doblar a la izquierda en 45 grados".

Diversas investigaciones indican que el uso de estrategias egocéntricas no implica ausencia de aprendizaje espacial (Dale, 1986; Dale e Innis, 1986; Einon, 1980; Foreman, 1985), aún cuando ambas formas de aprendizaje pueden estudiarse independiente en el laberinto radial (Dubreuil y cols., 2003; Olton y cols., 1977). Al parecer los animales en el laberinto radial basan su conducta en el uso simultáneo de varias estrategias y fuentes de información, que son complementarias en vez de excluyentes. Hallazgos similares han sido encontrados en el estudio de otras formas de comportamiento espacial, como la integración de vía (para revisiones, ver Etienne, Maurer y Seguinot, 1996).

El reconocimiento de que los roedores aprenden tanto a partir de claves visuales alocéntricas como de claves propioceptivas egocéntricas, y pueden utilizar estrategias basadas en los dos tipos de información, ha llevado a los investigadores a preguntar bajo qué condiciones se elige utilizar una u otra. En este sentido, se han identificado una serie de variables que influyen sobre la probabilidad de que los roedores utilicen estrategias egocéntricas en el laberinto de brazos radiales (e.g., Dale e Innis, 1986; Einon, 1980; Foreman, 1985; Hall y Berman, 1995; Hodges, 1996; Olton, Collison y Werz, 1977; Pico y Davis, 1984; Rossi-Arnaud, Fagioli, S. y Ammassari-Teule, 1991; Saavedra, Pinto, Marchant y Urzúa, 1999; Yoerg y Kamil, 1982). Se ha hipotetizado (e.g., Yoerg y Kamil, 1982; Saavedra y cols., 1999) que en muchos casos se privilegia el uso de estrategias egocéntricas por su funcionalidad, es decir, se debería al resultado de un análisis de costo-beneficio, producto del cual se privilegia la estrategia egocéntrica ya que requiere de un procesamiento menos complejo y permite un abastecimiento óptimo, en el menor tiempo posible y minimizando el número de errores.

Para analizar esta funcionalidad del uso de estrategias simplificadoras, una de las mejores opciones parece ser profundizar en el estudio de la influencia que el nivel de deprivación alimenticia tiene sobre la elección de una estrategia. Específicamente, en estudios previos se ha encontrado que las ratas con un mayor nivel de deprivación utilizan más la estrategia de uso del brazo adyacente que las ratas menos deprivadas (Saavedra y cols., 1999; Perry, com. pers. en Hodges, 1996). El uso de una estrategia simplificadora sería más frecuente con un nivel mayor de deprivación alimenticia porque representaría una forma de abastecerse del mayor monto posible de comida en el menor tiempo, lo que es necesario para asegurar la supervivencia bajo condiciones de escasez de comida. En la presente investigación nos hemos propuesto indagar con mayor profundidad en la forma en que el grado de deprivación alimenticia puede influir sobre el uso de estrategias egocéntricas en el laberinto radial, para lo cual nuestro primer objetivo ha sido intentar obtener los resultados que indican que tal relación existe, por medio de dos experimentos con procedimientos muy similares a los reportados por 
otros autores previamente (Saavedra y cols., 1999; Perry, com. pers. en Hodges, 1996).

\section{Experimento 1}

\section{Método}

Sujetos. Los sujetos fueron 24 ratas albinas macho sin experiencia previa en investigación de laboratorio, con una edad aproximada de 100 días y un peso de entre 300 y 390 gramos al comienzo de la investigación. Las ratas fueron puestas en cajas individuales con acceso libre a agua al momento de comenzar a ser registrado su peso de alimentación libre, para partir con la deprivación de comida luego de una semana. En este punto fueron aleatoriamente asignadas a dos grupos de 12 sujetos cada uno: G75 en el cual fueron deprivadas al $75 \%$ de su peso de alimentación libre, y G85 en el cual fueron deprivadas al 85\% de su peso de alimentación libre. El procedimiento de deprivación se detalla en la sección procedimientos.

Aparato. El aparato consistió en un laberinto radial elevado a $90 \mathrm{cms}$. desde el suelo, compuesto de ocho brazos idénticos de $70 \mathrm{x}$ 7,5 cms., unidos a una plataforma central con un diámetro de $30 \mathrm{cms}$ (ver fig. 1). El piso del laberinto y el pedestal sobre el cual se eleva son de madera pintada de color blanco, mientras sus muros están fabricados con Plexiglass transparente con $15 \mathrm{cms}$. de alto. En el extremo distal de cada brazo del laberinto se ubica un recipiente circular en el que se puede depositar el refuerzo para la rata. El aparato fue ubicado en una sala de experimentación con luz artificial y varias señales visuales ambientales (luces, puerta, experimentador, etc.). El refuerzo utilizado fueron granos de arroz inflado (Natur).

\section{Procedimiento}

Deprivación: Una semana antes del comienzo del entrenamiento las ratas fueron puestas bajo un régimen de deprivación alimenticia basado en un monitoreo del peso del animal.
Para ello, se registró previamente el peso de alimentación libre de cada rata, y durante el resto del experimento se mantuvo su peso en un $75 \%$ o un $85 \%$ del peso inicial, dependiendo de si pertenecían a los grupos G75 o G85, respectivamente. Para llevar a cabo dicha tarea el peso de cada rata fue monitoreado diariamente, aproximadamente a la misma hora, y se corrigieron desviaciones en el peso deseado modificando la ración de comida entregada para ese día en particular.

Exploración: Durante 3 días se dejó a cada rata explorar el laberinto individualmente por 10 minutos para adaptarlas a éste. No se presentó comida durante estas sesiones de exploración.

Fase de entrenamiento: En esta fase todos los sujetos recibieron una sesión diaria de entrenamiento durante 12 días. Al comienzo de cada sesión de entrenamiento se ubicó un refuerzo al final de cada brazo del laberinto. Dichos refuerzos no fueron reemplazados a lo largo de la sesión, por lo que cada sujeto tuvo acceso a un máximo de 8 piezas de comida. Al comienzo de la sesión, cada rata fue ubicada en la plataforma central del aparato, y la sesión se dio por finalizada al cumplirse una de las siguientes condiciones: (i) si el sujeto visitó los 8 brazos del laberinto, (ii) realizó 16 visitas, o (iii) se cumplieron 10 minutos desde el comienzo de la sesión. Estas condiciones han sido planteadas por los creadores del laberinto radial (Olton y Samuelson, 1976), y son comúnmente utilizadas en las investigaciones actuales con este aparato.

\section{Resultados y Discusión}

Los resultados relativos a la precisión de las ratas en la ejecución de la tarea se muestran en la figura 2. En el panel A se puede observar que las ratas comenzaron con una media en el porcentaje de errores de aproximadamente $30 \%$, la que disminuyó hasta llegar a un 6-9\% en las últimas sesiones, mejorando el rendimiento en ambos grupos a lo largo del entrenamiento. El porcentaje de 
errores promedio total para G75 fue de $14.47 \%$, y para el G85 de $17.12 \%$, diferencia que no resultó significativa según una prueba $\mathrm{T}$ de Student para varianzas iguales ( $\mathrm{p}>0.1, \mathrm{~T}=0.71, \mathrm{gl}=22$ ). Resultados distintos se observan en el panel B de la figura 2, el que muestra el tiempo de finalización de la tarea promedio por grupo a lo largo del entrenamiento. Se puede observar que en ambos grupos disminuye el tiempo de finalización desde sobre 350 segundos en la primera sesión hasta entre 100 y 150 segundos en las sesiones finales, disminución que es más rápida en el G75 (bajando de 150 segundos ya en el segundo día de entrenamiento) que en el G85 (el que sólo a partir de la séptima sesión logró un tiempo de finalización promedio menor que 150 segundos). Una prueba $\mathrm{T}$ de Student para muestras independientes con varianzas iguales ha confirmado la significación estadística de esta diferencia $(\mathrm{p}<0.05, \mathrm{~T}=2.13, \mathrm{gl}=22)$, lo que es evidencia indirecta de que el método de deprivación utilizado efectivamente logró un mayor nivel de motivación para el G75 que para el G85, aún cuando la precisión en la discriminación no haya sido afectada diferencialmente para ambos grupos.
Figura 2: Comparación dela precisión de los grupos G75 y G85 a lo largo de las 12 sesi ones de entrenamiento del experimento 1. Pane $A$ : porcentaje de errores promedi o para ambos grupos a lo largo del entrenamiento. Panel B: tiempo de fi nalización dela tarea promedi o para ambos grupos a lo largo del entrenamiento.

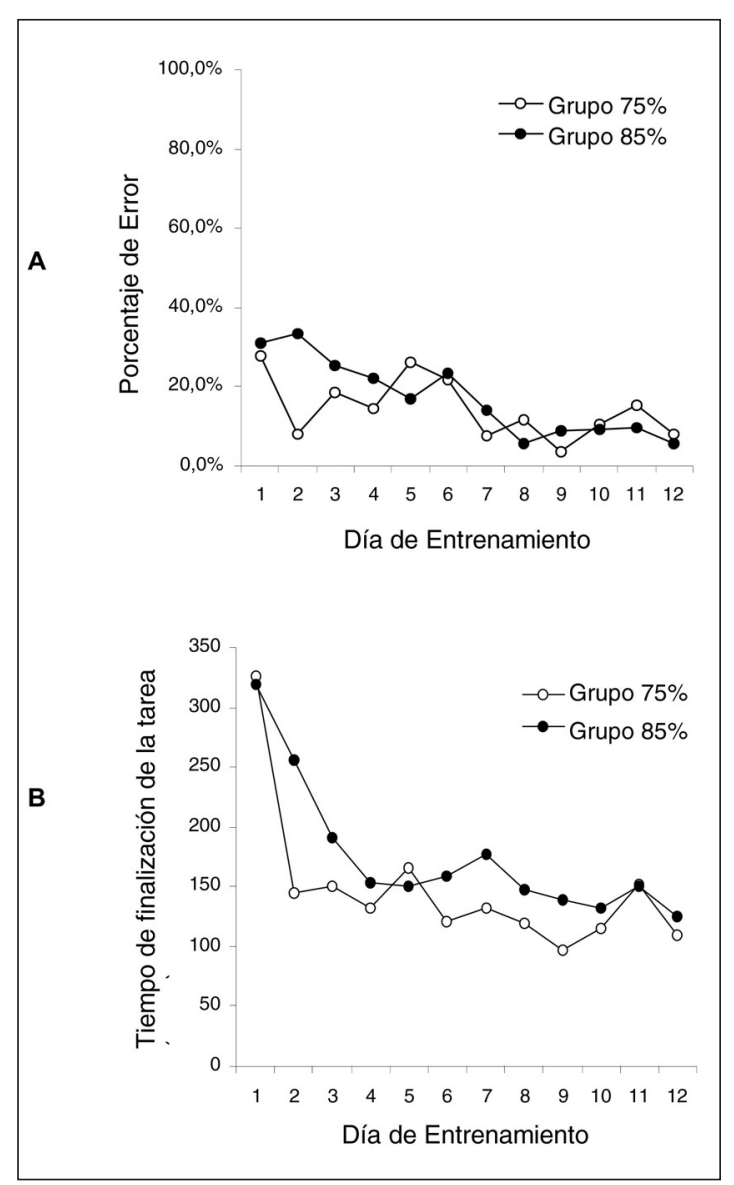

Los resultados más importantes para el presente experimento se presentan en la figura 3, la que muestra la frecuencia de uso del brazo adyacente (F.U.B.A.) para ambos grupos. El G75 muestra un porcentaje de F.U.B.A. promedio de $56.34 \%$, mientras tal medida en el G85 alcanza un valor de 59.89\%, encontrándose una diferencia que no alcanza significación estadística según una prueba $\mathrm{T}$ de Student para muestras independientes y varianzas iguales $(p>0.1, T=0.79, g l=22)$. Estos resultados resultan inesperados, debido a que son contradictorios con los obtenidos previamente en otras investigaciones (Saavedra 
y cols., 1999; Perry, com. pers. citada por Hodges, 1996), las que sí encontraron diferencias significativas en la F.U.B.A. para grupos deprivados de comida diferencialmente.

Figura 3: Comparación del porcentaje medio de F.U.B.A. para los grupos G75 y G85, cal culado para todas las sesiones de entrenamiento del experimento 1. Las barras de error representan $\mathrm{d}$ error típico de la media.

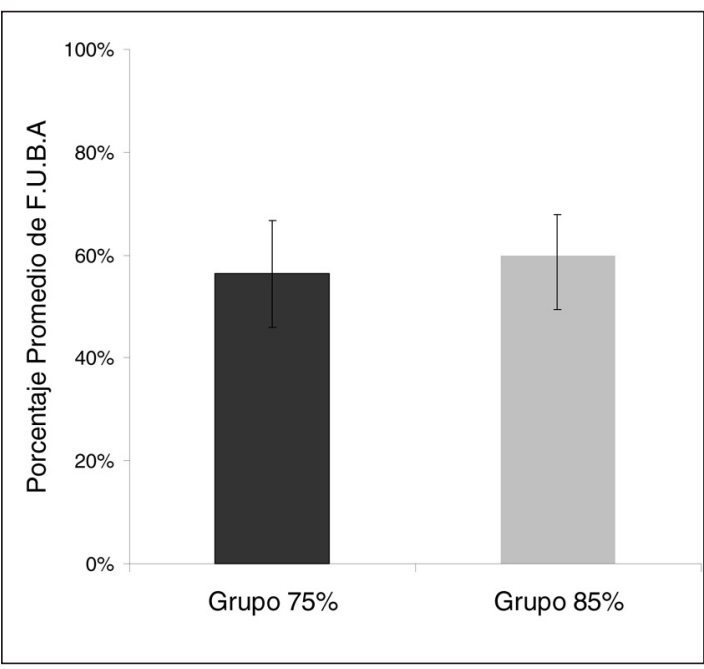

Ahora bien, los resultados obtenidos en el presente experimento no pueden ser tomados apresuradamente como evidencia de que no existe la relación informada por otros autores entre el nivel de deprivación alimenticia y el uso de estrategias egocéntricas en el laberinto radial. Esto principalmente porque, desde el punto de vista estadístico, no podemos controlar la probabilidad de error para llegar a tal conclusión (error tipo II), mientras que en las investigaciones previas se ha llegado a conclusiones contrarias con una probabilidad de error considerablemente pequeña (error tipo I). Es por esto que, para entregar mayor generalidad a nuestros resultados, hemos realizado un segundo experimento similar al presente, pero con pequeñas modificaciones.

En primer lugar, se ha cambiado el método de deprivación de los animales debido a que el método utilizado en el experimento 1 , si bien es exactamente igual al utilizado previamente en la investigación de Saavedra y colegas (1999), implicó un solapamiento de las raciones diarias de comida para los sujetos en los G75 y G85, debido a que se encontraba basado en un monitoreo del peso del animal y no en una ración diaria fija de alimento para cada sujeto. En el experimento 2, por tanto, el método de deprivación ha sido modificado para hacerlo similar al utilizado por Perry (com. pers. en Hodges, 1996), quien también ha encontrado una mayor uso de estrategias egocéntricas para ratas más motivadas.

Por otro lado, el procedimiento utilizado durante el presente experimento difiere levemente del utilizado en investigaciones previas (Saavedra y cols., 1999) en donde sí se presentó refuerzo a los sujetos durante la fase de exploración del laberinto. Si bien parece poco probable a primera vista, tal vez dicha diferencia puede haber influido en nuestros resultados, de modo que el experimento 2 incluye también el refuerzo durante la exploración.

\section{Experimento 2}

\section{Método}

Sujetos. Los sujetos fueron 9 ratas albinas macho con características similares a las del experimento 1. Las ratas fueron aleatoriamente asignadas a dos grupos: un grupo al que se asignó una ración diaria de alimento baja (GB), con 5 sujetos, y otro grupo con una ración alta (GA), con 4 sujetos. El procedimiento de deprivación se detalla en la sección procedimientos.

Aparato. El aparato fue el mismo que en el experimento 1.

Procedimiento. El procedimiento fue exactamente igual al del experimento 1, excepto por dos aspectos. En primer lugar, el método de deprivación basado en el monitoreo del peso de los animales, utilizado en el experimento 1, fue cambiado por un método 
de deprivación basado en raciones fijas diarias de alimento. De este modo, a los sujetos en el GB se les entregó por igual una ración diaria de 10 gramos de comida, mientras para los sujetos en el GA la ración diaria fue de 20 gramos. Por datos previamente recogidos en nuestro laboratorio, sabemos que las ratas de entre 90 y 120 días de edad con raciones de comida de 10 gramos se mantienen aproximadamente en un $70 \%$ de su peso de alimentación libre, mientras que con una ración de 20 gramos se mantienen aproximadamente en un $90 \%$ de su peso de alimentación libre. Las ratas de esta edad en régimen de alimentación libre comen diariamente una media (aprox.) de 30 gramos.

La segunda diferencia con respecto al procedimiento del experimento 1 radica en que durante la fase de exploración sí se presentaron refuerzos, los que se dispersaron por todo el laberinto para motivar a las ratas a recorrerlo. Esta modificación en el procedimiento se llevó a cabo por dos razones: en primer lugar, se notó que en el experimento de Saavedra y colegas (1999), en el que se encontraron diferencias en la frecuencia de utilización del brazo adyacente (F.U.B.A) según el nivel de deprivación, sí se había presentado comida durante la fase de exploración, por lo que esta diferencia podría estar influyendo sobre nuestros resultados en el experimento 1; y en segundo lugar, durante el primer experimento algunos sujetos no comenzaron a explorar correctamente el laberinto desde las primeras sesiones, problema que no surge cuando se distribuye comida durante la exploración.

\section{Resultados y Discusión}

Tal como se puede observar en la figura 4-A, las ratas en ambos grupos lograron una ejecución bastante precisa en la tarea desde los primeros días de entrenamiento, manteniendo dicha precisión a lo largo del experimento. El porcentaje de errores promedio a lo largo del entrenamiento para el grupo B fue de $12.89 \%$, y para el grupo A de $12.42 \%$, con una pequeña diferencia que no resulta significativa al ser analizada mediante un $\mathrm{T}$ de Student para varianzas iguales ( $p>0.1$, $\mathrm{T}=0.13, \mathrm{gl}=7)$. El análisis gráfico del tiempo de finalización de la tarea (ver figura 4-B) indica una mejora en el desempeño de las ratas a lo largo del entrenamiento, y tampoco se encuentra una diferencia significativa entre ambos grupos con respecto a este indicador según la prueba $\mathrm{T}$ de Student para varianzas iguales ( $p>0.1, \mathrm{~T}=0.47, \mathrm{gl}=7)$, a diferencia de lo ocurrido en el experimento 1.

Figura 4: Comparación dela precisión de los grupos A y B a lo largo de las 11 sesiones de entrenami ento de experimento 2 . Panel $A$ : porcentaje de errores promedio para ambos grupos a lo largo del entrenamiento. Panel B: tiempo de finalización de la tarea promedi o para ambos grupos a lo largo del entrenamiento.

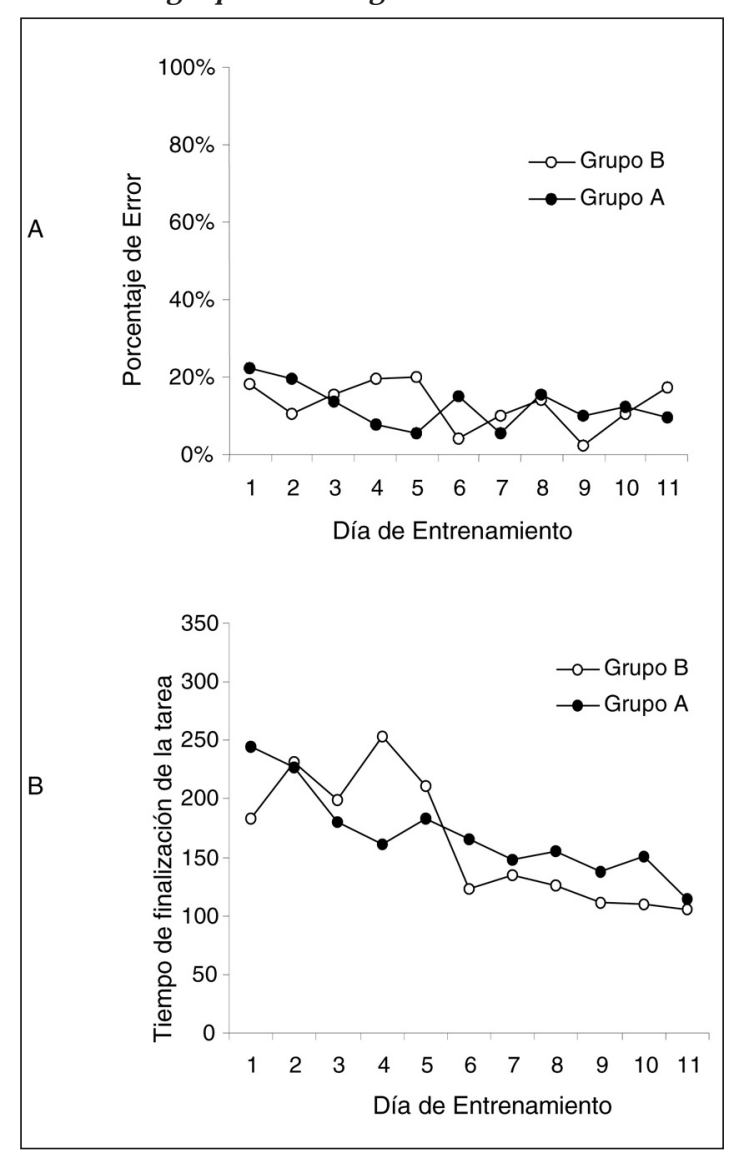


Los datos de mayor relevancia de este experimento se muestran en la figura 5. Confirmando los resultados del experimento 1, la figura muestra claramente que no se observaron diferencias entre ambos grupos con respecto a la F.U.B.A., con los sujetos en el grupo B realizando en promedio a lo largo del entrenamiento un $55.13 \%$ de sus elecciones en el brazo adyacente, lo que es bastante similar a la media de $59.10 \%$ calculada para el grupo A. Estos resultados han sido confirmados por el análisis estadístico mediante una prueba $\mathrm{T}$ de Student para varianzas iguales $(\mathrm{p}>0.1, \mathrm{~T}=0.27, \mathrm{gl}=7)$.

Figura 5: Comparación del porcentaje medio de

F.U.B.A. para los grupos B y A, calculado para todas las sesi ones de entrenamiento del experimento 2. Las barras de error representan el error típico de la media.

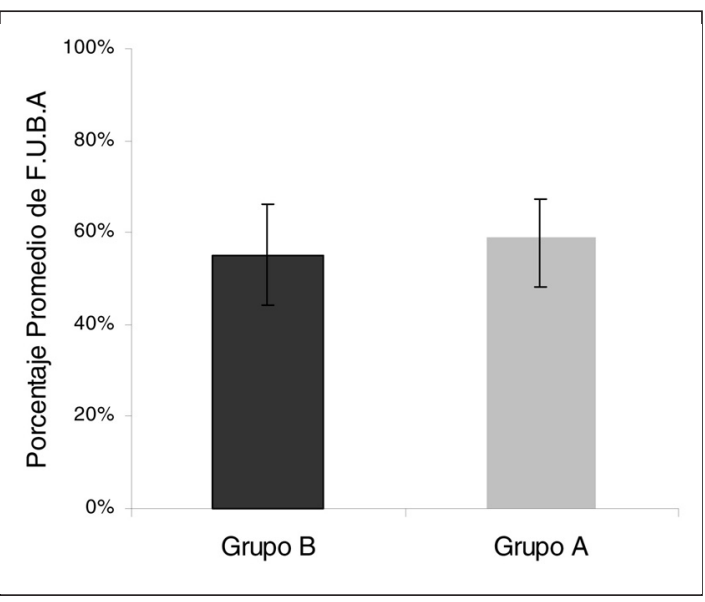

Nuevamente hemos sido incapaces de reproducir los resultados obtenidos previamente en otros laboratorios (Saavedra y cols., 1999; Perry, com. pers. en Hodges, 1996), y que sugieren una relación entre el nivel de deprivación de comida en ratas y el grado hasta el cuál éstas utilizan estrategias egocéntricas de respuesta en el laberinto radial. Los resultados del experimento 2 confirman aquellos del experimento 1, y entregan mayor evidencia para argumentar que, al menos bajo ciertas circunstancias, tal relación podría no cumplirse. Con esto aumenta además la generalidad de los hallazgos del experimento 1 , dado que no se ha encontrado diferencia alguna en los resultados para ambos grupos aún cuando se ha utilizado un método de deprivación alimenticia distinto al usado previamente y similar al que ha desarrollado Perry (com. pers. en Hodges, 1996), y también se ha variado el procedimiento durante la fase de exploración, haciéndolo similar al utilizado por Saavedra y colegas (1999).

Sin embargo, debemos conservar la cautela con nuestras conclusiones, debido principalmente a que los resultados aquí expuestos, desde el punto de vista estadístico, no nos permiten aceptar una hipótesis nula sin un considerable margen de error no controlado, y por tales razones no pueden competir con la fuerza estadística de resultados previamente documentados en que sí se han encontrado diferencias significativas en la F.U.B.A. bajo distintas condiciones de deprivación. A nuestro favor tenemos que los resultados de los experimentos 1 y 2 son bastante similares, de modo tal que la generalidad de éstos, que no puede comprobarse mediante pruebas estadísticas, sí se consigue mediante su obtención repetida. A continuación se discuten las implicaciones que pueden tener nuestros resultados, y se ofrecen posibles soluciones a la aparente contradicción con resultados previamente publicados.

\section{Discusión General}

En dos experimentos, hemos intentado infructuosamente entregar evidencia de una relación entre el nivel de deprivación alimenticia y el uso de estrategias egocéntricas por ratas en el laberinto radial. A pesar de que los resultados de ambos experimentos son nulos, se complementan para dar cierta generalidad a la conclusión de que, al menos bajo ciertas condiciones, no es posible observar tal relación. Por otro lado, puesto que en otras investigaciones existen hallazgos contradictorios con los presentes resultados, es probable que la relación entre ambas variables sea en realidad más compleja de lo que hasta ahora se suponía. 
Una hipótesis que podría explicar estos resultados contradictorios sería que la relación entre nivel de deprivación alimenticia y uso de estrategias egocéntricas de respuesta existe, pero se encuentra modelada por variables actualmente desconocidas. Los resultados obtenidos en los presentes experimentos implican que probablemente no se encuentran entre tales variables ni el método de deprivación utilizado, ni la presencia de comida en el laberinto durante la fase de exploración, ya que ambas variables han sido manipuladas diferencialmente en los dos experimentos, con iguales resultados (excepto por una mejora en el rendimiento de los sujetos durante los primeros días de entrenamiento en el experimento 2 , probablemente debido a la disponibilidad previa de refuerzo en la fase de exploración). Por otro lado, la cepa de los sujetos ha sido manipulada por Saavedra y colegas (1999) con resultados similares para ratas albinas, hooded y grises, mientras el aparato y los sujetos utilizados han tenido iguales características en los presentes experimentos y en los de investigaciones previas. Aún así, es posible que diferencias en el número y la calidad de las claves visuales distales disponibles en la sala de experimentación hayan influido sobre los resultados de cada investigación. Por ejemplo, una sala de experimentación con menos claves ambientales podría llevar al uso consistente de estrategias egocéntricas, sin importar el nivel de deprivación de las ratas. Otra diferencia con las investigaciones previas radica en que durante los experimentos aquí reportados los sujetos fueron mantenidos en cajas individuales, mientras en la investigación de Saavedra y colegas (1999) se mantuvieron en grupos de 3-4 ratas por caja. Puesto que en las cajas grupales existe suficiente espacio en el comedero como para que todas las ratas se alimenten simultáneamente, parece poco probable que este último factor sea el responsable de las diferencias en los resultados.

Una segunda hipótesis es que la relación entre deprivación y uso de estrategias de búsqueda de comida en el laberinto radial no sea simplemente lineal, sino que dependa de cierto "umbral" de deprivación, de modo tal que las ratas utilizan en mayor medida una estrategia estereotípica solamente una vez que han sobrepasado este umbral. Desde este punto de vista, tal vez los procedimientos en nuestros experimentos no han logrado deprivar a las ratas suficientemente como para encontrar un mayor uso de estrategias egocéntricas, lo que sí se habría logrado en investigaciones previas. Sin embargo, esta explicación resulta poco verosímil debido a que las ratas en el G75 del experimento 1 fueron mantenidas bajo un $75 \%$ de su peso de alimentación libre, llegando a un promedio de aproximadamente $70 \%$ de dicho peso durante el entrenamiento, un nivel tan bajo que puede implicar peligro de enfermedades, y que se encuentra bajo el peso promedio de las ratas en el experimento previo de Saavedra y colegas (1999).

Queda por ser investigado si estas u otras hipótesis pueden dar cuenta de la diferencia de los presentes resultados con los de otras investigaciones, pero sí puede concluirse que nuestros resultados desafían la hipótesis planteada previamente (Yoerg y Kamil, 1982; Saavedra y cols., 1999) que indica que las estrategias egocéntricas mostradas por animales en el laberinto radial serían resultado de un proceso de evaluación de costo-beneficio. Según esta hipótesis, derivada de la teoría del forrajeo óptimo, cuando los animales tienen acceso a varias estrategias de recolección de comida (como es el caso del laberinto radial) escogerán aquélla que sea más "económica" en términos de tiempo, procesamiento cognitivo, esfuerzo, etcétera, lo que además ocurre con mayor probabilidad en situaciones en que la comida es particularmente escasa. Los presentes resultados son un desafío para esta hipótesis en la medida en que de ser el uso de estrategias egocéntricas resultado de un proceso de optimización acentuado en circunstancias de escasez de comida, siempre habría diferencias en la 
F.U.B.A. al haber diferencias en el nivel de deprivación alimenticia, lo que no se encontró en nuestros experimentos. Las soluciones a este y otros problemas planteados por nuestros resultados requieren simplemente de mayor investigación empírica.

\section{Agradecimientos}

La presente investigación ha sido llevada a cabo gracias al apoyo de la Escuela de Psicología de la Universidad La República. Los autores desean agradecer especialmente a don Isidoro Neves, doña Carmen Contreras y don César Cañas.

\section{Referencias}

Ammassari-Teule, M., y CAprioli, A. (1985). Spatial learning and memory, maze running strategies and cholinergic mechanisms in two inbred strains of mice. Behavioural Brain Research, 17: 9-16.

Bennett, A. (1996). Do animals have cognitive maps? The Journal of Experimental Biology, 199: 219-224.

Chamizo, V. (2002). Spatial learning: Conditions and basic effects. Psicológica, 23: 33-57.

Chamizo, V. (2003). Acquisition of knowledge about spatial location: Assessing the generality of the mechanism of learning. The Quarterly Journal of Experimental Psychology, 56B: 102-113.

Dale, R. (1986). Spatial and temporal response patterns on the eight-arm radial maze. Physiology and Behavior, 36: 787-790.

Dale, R., e InNis, N. (1986). Interactions between response stereotypy and memory strategies on the eight-arm radial maze. Behavioural Brain Research, 19: 17-25.

Dubreuil, D., Tixier, C., Dutrieux, G., y Edeline, J. (2003). Does the radial arm maze necessarily test spatial memory? Neurobiology of Learning and Memory, 79: 109-117.

ECKERMAN, D. (1980). Monte Carlo estimatin of chance performance for the radial arm maze. Bulletin of the Psychonomic Society, 15: 93-95.

EinON, D. (1980). Spatial memory and response strategies in rats: Age, sex and rearing differences in performance. The Quarterly Journal of Experimental Psychology, 32: 473-489.

Etienne, A., Maurer, R., y Seguinot, V. (1996). Path integration in mammals and its interaction with visual landmarks. The Journal of Experimental Biology, 199: 201-209.

FOREMAN, N. (1985). Algorithmic responding on the radial maze in rats does not always imply absence of spatial encoding. The Quarterly Journal of Experimental Psychology, 37B: 333-358.

ForemAn, N., y STEVENS, R. (1982). Visual lesions and radial maze performance in rats. Behavioral and Neural Biology, 36: 126-136.

Gallistel, C. (1993): The Organization of Learning. Cambridge: MIT Press.

Good, M. (2002). Spatial memory and hippocampal function: Where are we now? Psicológica, 23: 109-138.

Hall, J., y Berman, R. (1995). Juvenile experience alters strategies used to solve the radial arm maze in rats. Psychobiology, 23: 195-198.

Hodges, H. (1996). Maze procedures: the radial arm and water maze compared. Cognitive Brain Research, 3: 167-181.

MacPhail, E. M. (2002). The role of the avian hippocampus in spatial memory. Psicológica, 23: 93-108. 
Olton, D., Collison, C., y Werz, M. A. (1977). Spatial memory and radial arm maze performance of rats. Learning and Motivation, 8: 289-314.

Olton, D., y Samuelson, R. (1976). Remembrance of places passed: Spatial memory in rats. Journal of Experimental Psychology: Animal Behavior Processes, 2: 97-116.

Pico, R., y DAVIS, J. (1984): The radial maze performance of mice: Assessing the dimensional requirements for serial order memory in animals. Behavioural and Neural Biology, 40: 5-26.

Poucet, B., y Benhamou, S. (1997). The neuropsychology of spatial cognition in the rat. Critical Reviews in Neurobiology, 11: 101-120.
Rossi-Arnaud, C., Fagioli, S., y AmmassariTeule, M. (1991). Spatial learning in two inbred strains of mice: Genotypedependent effect of amygdaloid and hippocampal lesions. Behavioural Brain Research, 45: 9-16.

Saavedra, M. A., Pinto, T., Marchant, F., Y URzÚA, C. (1999). Motivación: su efecto sobre la estrategia de solución del laberinto octogonal. Revista de Psicología de la Universidad de Chile, 8: 143-150.

Watts, J., Stevens, R., y Robinson, C. (1981): Effects of scopolamine on radial maze performance in rats. Physiology and Behavior, 26: 845-851.

Yoerg, S., y Kamil, A. (1982). Response strategies in the radial arm maze: Running around in circles. Animal Learning and Behavior, 10: 530-534. 
